01

\title{
Метод расчета термостата для наземных испытаний инфракрасных оптико-электронных систем
}

\author{
(ㄱ И.Ю. Дмитриев, А.А. Котмакова, Ю.А. Резунков
}

АО „Научно-исследовательский институт оптико-электронного приборостроения“, 188540 Сосновый Бор, Россия

e-mail: yuri@sbor.net

Поступило в Редакцию 2 июня 2020 г.

В окончательной редакции 13 июля 2020 г.

Принято к публикации 6 августа 2020 г.

Создана модель термовакуумной системы (термостата) для регулирования и стабилизации температуры главного зеркала охлаждаемого оптического коллиматора для проведения испытаний оптико-электронной аппаратуры, работающей в инфракрасной области. Разработан оригинальный метод решения системы нелинейных уравнений лучистого теплообмена в термостате, проведен анализ однородности распределения температуры по поверхности главного зеркала.

Ключевые слова: термостат, коллиматор, лучистый теплообмен, тепловой баланс, термоаберрации.

DOI: 10.21883/JTF.2021.02.50354.190-20

\section{Введение}

Испытания инфракрасной (ИК) оптико-электронной аппаратуры (ОЭА) с охлаждаемой оптикой, работающей в режиме ограничения фона, проходят в условиях вакуума и низкого радиационного фона. Последнее достигается охлаждением оптических элементов коллиматора и исключением прямой засветки входного зрачка ИК ОЭА „теплыми“ элементами вакуумной камеры.

Разработка системы температурного регулирования и стабилизации уровня внутреннего радиационного фона требует создания специальных расчетных методов теплофизического анализа сложных технических систем, состоящих из оптического и криогенно-вакуумного оборудования. В состав термовакуумного стенда, как правило, входит вакуумная камера большого объема $\left(150 \mathrm{~m}^{3}\right.$ и более) с давлением остаточного воздуха до $10^{-7}$ Torr, a также система терморегулирования с набором охлаждаемых экранов, оборудование для установки испытуемого изделия, элементы коллиматора, включая главное зеркало коллиматора (ГЗК) и фокальный узел для имитации объектов наблюдения [1].

Оценка пространственного и энергетического разрешения ИК ОЭА с охлаждаемой оптикой требует высококачественного коллиматора, не расстраиваемого в большом диапазоне низких температур. Особенно это относится к ключевому элементу коллиматора - ГЗК. Охлаждение элементов коллиматора до рабочих температур при сохранении высокого оптического качества изображения имитатора объекта наблюдения (близкого к дифракционному) - задача, которая рассматривается с учетом ряда технических требований, а именно:

- система температурного регулирования должна обеспечить стабилизацию низкотемпературного радиационного фона в поле обзора испытуемого изделия;
— термоаберрации зеркал коллиматора должны быть минимальными;

- максимальная локализация охлаждаемого объема с учетом ограниченных возможностей криогенной системы испытательного стенда.

В основе предлагаемого метода терморегулирования и стабилизации внутреннего радиационного фона рассматривается лучистый (бесконтактный) способ охлаждения оптики, т. е. за счет обмена тепловым излучением между элементами коллиматора и специальными охлаждаемыми экранами, которые при этом не должны ограничивать доступ к оптическим элементам при их юстировке и эксплуатации.

Радиационный теплообмен связан с двойным преобразованием энергии: первоначально внутренняя энергия тела превращается в энергию электромагнитного излучения, а затем, после переноса энергии в пространстве электромагнитными волнами происходит переход лучистой энергии во внутреннюю энергию другого тела. Известно [2], что лучистый теплообмен между поверхностями твердых тел зависит от их температуры (в 4 степени в соответствие с законом Стефана-Больцмана), радиационных свойств этих поверхностей (характеризуемые степенью черноты) и от „геометрии“ теплового взаимодействия, т. е. от геометрических форм этих тел. Однако эффективная температура радиационного фона на входе в испытуемое изделие в той или иной степени может превышать термодинамическую температуру элементов коллиматора [3] из-за дополнительных тепловых потоков от стенок вакуумной камеры.

Глубокое охлаждение радиационного фона в [3] было реализовано за счет полной изоляции внутренних стенок камеры охлаждаемыми экранами, что при большом объеме камеры представляется сложным и требует значительных материальных затрат как при ее создании, так и при эксплуатации испытательного стенда. 
В настоящей работе проблемы терморегулирования и стабилизации внутреннего радиационного фона в коллиматоре испытательного стенда предлагается решать на примере охлаждения ГЗК с помощью термовакуумной системы - термостата, обеспечивающего также минимизацию температурных деформаций ГЗК. С этой целью создана теплофизическая модель „ГЗК-термостат“ в виде системы уравнений лучистого теплообмена в замкнутых системах и предлагается итерационный метод их решения с привлечением стандартных компьютерных программ. Анализ полученных результатов позволяет сформулировать рекомендации по развитию метода стабилизации на основе термостата к проектированию термовакуумных стендов.

\section{1. Теплофизическая модель термовакуумной системы температурной стабилизации}

В системах терморегулирования и стабилизации низкотемпературного радиационного фона в замкнутых пространствах в вакууме $[1,3,4]$ можно выделить три принципиальных с теплофизической точки зрения объекта [5], а именно:

- тепловые источники, имеющие внешний подвод тепла, и стоки - объекты с внешним индивидуальным криогенным охлаждением; к тепловым источникам можно отнести стенки вакуумной камеры, а к тепловым стокам - охлаждаемые экраны;

- теплозащитные экраны и т.П., используемые для уменьшения теплового потока от тепловых источников;

- базовые элементы (в данном случае - элементы коллиматора), которые являются объектами охлаждения и температурной стабилизации.

В настоящей работе система температурной стабилизации (термостат) представляется в виде отдельных блоков, в каждом из которых есть свой базовый элемент, а процесс температурной стабилизации можно рассматривать отдельно для каждого блока. На рис. 1 приведена функциональная схема термостата, элементы которого 2-4 и 6 расположены внутри цилиндрической вакуумной камеры 5 испытательного стенда. Главное зеркало коллиматора 1 с подставкой 8 (базовый элемент) расположено внутри термостата. Принципиальной особенностью термостата является охлаждение оптических элементов коллиматора до заданной температуры $T_{1}$ за счет лучистого теплообмена с двумя осесимметричными (относительно оси зеркала) цилиндрическими экранами 2 и 3 (тепловые стоки) и дополнительного экрана 4, используемого для стабилизации теплового баланса с торцевой стороны зеркала. Внутренний диаметр охлаждаемых экранов выбирается из условия максимальной эффективности теплового обмена между ними и зеркалом (см. далее). Геометрические параметры цилиндрических охлаждаемых экранов ограничены условиями исключения виньетирования световой зоны коллиматора. Охлаждение экранов осуществляется внешней криогенной системой таким образом, чтобы температуры этих элементов $T_{2}, T_{3}$ и $T_{4}$ соответствовали условиям теплового баланса в системе „ГЗК-термостат“. Температура стенок вакуумной камеры (тепловой источник) $T_{5}$ считается постоянной и равной температуре окружающего воздуха. Для уменьшения теплового потока со стороны стенок вакуумной камеры на зеркало и охлаждаемые экраны используются специальные цилиндрические теплозащитные экраны 6, устанавливаемые между ними. Зеркало с подставкой устанавливается на оптический стол 9, с которым существует непосредственный тепловой контакт.

Как видно из представленной схемы, теплофизическая модель термостата должна включать в себя лучистый теплообмен между собой большого количества элементов. Кроме этого, часть из них, например, главное зеркало коллиматора с подставкой, находятся в непосредственном тепловом контакте, что приводит к необходимости учета теплопередачи между ними за счет теплопроводности материалов, из которых состоят эти элементы. В теплофизической модели следует также учитывать разнообразие геометрических, теплофизических и оптических свойств материалов элементов термостата и ГЗК $[6,7]$.

Возможность достижения теплового равновесия (или баланса тепловых потоков) в термовакуумной системе рассматривается нами на основе вариационного принципа для замкнутой системы при всех возможных изменениях внутреннего состояния, не влияющих на ее энтропию; вариация тепловой энергии исчезала или была положительной [8]. В случае только лучистого теплообмена энергией $i$-го элемента с окружающими его телами состояние равновесия может быть представлено в следующем виде:

$$
\sum_{j} P_{i j} \delta T_{j}=0
$$

где $\left[P_{j}\right]=W / K, j-$ номер окружающего элемента. Параметры $P_{i j}$ соответствуют мощности лучистого потока при изменении температуры $i$-го элемента на $1 \mathrm{~K}$. В случае дополнительного учета кондуктивной теплопроводности между $i$-м и $n$-ми элементами условие (1) можно представить в виде

$$
\sum_{j} P_{i j} \delta T_{j}+\sum_{n} J_{i n} \delta T_{n}=0,
$$

где $n$ - номер элемента, от которого тепло передается кондуктивным способом. Параметр $J_{\text {in }}$ характеризует тепловую мощность, передаваемую кондуктивным способом при температурной разнице в $1 \mathrm{~K}$.

Для главного зеркала (коллиматора) уравнение теплового баланса означает, что сумма обмена тепловых потоков с элементами, участвующими в лучистом теп- 


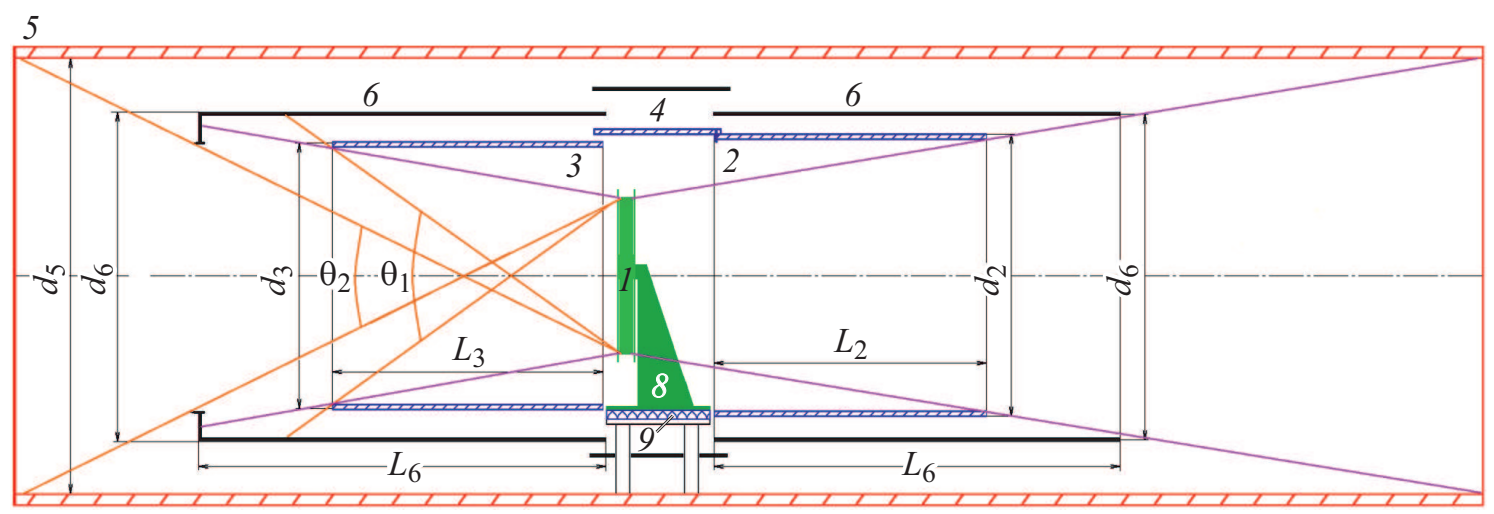

Рис. 1. Структурная схема модели термостата. $1-$ ГЗК, $2-4,6-$ экраны, $5-$ вакуумная камера, $8-$ подставка, $9-$ оптический стол.

лообмене с зеркалом, равна нулю, т.е.

$$
\sum_{i=2}^{N} \varepsilon_{1 i} \sigma_{0} \varphi_{i 1} F_{i}\left(T_{i}^{4}-T_{1}^{4}\right)=0,
$$

где $N$ - количество элементов термостата, связанных лучистым теплообменом с главным зеркалом, $\varepsilon_{1 i}-$ приведенные степени черноты соответствующих пар элементов (зеркало 1 и $i$-ый элемент), $\sigma_{0}$ - постоянная Стефана-Больцмана, $\sigma_{0}=5.67 \cdot 10^{-8} \mathrm{~W} /\left(\mathrm{m}^{2} \cdot \mathrm{K}^{4}\right)$, $\varphi_{i 1}$ - угловые коэффициенты теплового излучения [2], которые показывают, какая доля лучистого теплового потока попадает с $i$-го элемента на главное зеркало 1 , $F_{i}$ - площадь излучающей поверхности $i$-го элемента, $T_{i}$ - температура $i$-го элемента, $T_{1}$ - температура главного зеркала коллиматора. Здесь [6]

$$
\varepsilon_{i j}=\frac{1}{1+\varphi_{i j}\left(\frac{1}{\varepsilon_{i}}-1\right)+\varphi_{j i}\left(\frac{1}{\varepsilon_{j}}-1\right)} \text { и } \varphi_{j i}=\frac{F_{i}}{F_{j}} \varphi_{i j},
$$

где $\varepsilon_{i}$ и $\varepsilon_{j}-$ степени черноты поверхности излучающего и принимающего излучение твердых тел.

Если между некоторыми элементами рассматриваемой системы существует дополнительный тепловой контакт, то суммарный поток обмена тепловой энергии между ними по аналогии с (2) может быть описан следующей формулой:

$$
Q_{i}=\sum_{j} \sigma_{0} \varepsilon_{i j} \varphi_{j i} F_{j}\left(T_{j}^{4}-T_{i}^{4}\right)+\sum_{n} \frac{\lambda_{n}}{\delta_{n}} F_{n}\left(T_{n}-T_{i}\right)=0,
$$

где $\delta_{n}-$ геометрическая толщина элемента, через который передается тепло из-за теплопроводности материала.

В случае прямого контакта в качестве тепловых граничных условий используются условия 4-го рода, когда задается равенство температуры и тепловых потоков на границе раздела элементов $i$ иj:

$$
T_{i}=T_{j} \quad \text { и } \quad \lambda_{i} \frac{\partial T_{i}}{\partial n}=\lambda_{j} \frac{\partial T_{j}}{\partial n},
$$

где $\lambda_{i}, \lambda_{j}-$ коэффициенты теплопроводности материалов соответствующих элементов.

\section{2. Метод расчета температурных параметров термостата}

Воспользуемся уравнениями типа (5) для расчета температурных параметров элементов термостата при заданном значении температуры зеркала коллиматора. В общем случае в термооптической системе стабилизации (термостате) имеется $N=n+m+k$ элементов, из которых $n$ - суммарное количество тепловых источников $\left(n_{i}\right)$ и стоков $\left(n_{s}\right), m-$ количество теплозащитных экранов и $k-$ количество базовых элементов. В соответствии с постановкой задачи для всех элементов, кроме тепловых источников и стоков, записываются уравнения теплового баланса. В результате имеется $m+k$ уравнений $(5)$ и $\left(m+n_{s}\right)$ неизвестных. Требуемые значения температуры базовых элементов и источников тепла считаются известными, т.е. система содержит $\left(k+n_{i}\right)$ известных величин. Для получения замкнутой системы уравнений требуется привлечение еще $\left(n_{s}-k\right)$ уравнений. В случае конкретной схемы термостата (рис. 1 ), когда $n_{s}=2$, a $k=1$, не хватает еще одного уравнения. Эта особенность системы уравнений на основе (5) позволяет ее дополнить, используя тот факт, что при охлаждении коллиматора необходимо обеспечить минимальные аберрации зеркала, вызванные градиентами температуры в нем. Для минимизации температурных градиентов введем условие равенства тепловых потоков на поверхность зеркала с двух его сторон (т.е. слева и справа на рис. 1). Это позволит исключить появление градиентов температуры по оси зеркала и соответственно минимизировать термоискажения его поверхностей из-за большой толщины зеркала.

В такой постановке задача сводится к нахождению решения системы из $\left(m+n_{s}\right)$ нелинейных (4-ой степени) алгебраических уравнений, в которых температура зеркала коллиматора задается постоянной и равной $T_{1}$, и требуется определить температуры охлаждаемых экранов.

Для решения исходной системы уравнений запишем уравнения теплового баланса типа (5) в соответствии с вариационным принципом в виде (2), где примем 
$\delta T_{i}=T_{i}-T_{1}$. Т. е. в установившемся тепловом равновесии термовакуумной системы в целом температура каждого элемента будет определяться температурой зеркала коллиматора $T_{1}$, которая задает необходимый радиационный фон. В этом случае, например, уравнение теплового баланса для зеркала преобразуется к виду

$$
\sum_{i=1}^{N} \varepsilon_{1 i} \sigma_{0} \varphi_{i 1} F_{i}\left(T_{i}^{2}+T_{1}^{2}\right)\left(T_{i}+T_{1}\right) \delta T_{i}+\sum_{j}^{n} \frac{\lambda_{j}}{\delta_{j}} F_{j} \delta T_{j}=0 .
$$

Аналогично преобразуются и все $\left(m+n_{s}\right)$ алгебраических уравнений. Конкретный вид каждого уравнения из системы можно проиллюстрировать следующим примером, в котором представлены оба варианта уравнения теплового баланса главного зеркала.

Начальное уравнение имеет следующий вид:

$$
\begin{aligned}
& \varepsilon_{12} \sigma_{0} \varphi_{21} F_{2}\left(T_{2}^{4}-T_{1}^{4}\right)+\varepsilon_{13} \sigma_{0} \varphi_{31} F_{3}\left(T_{3}^{4}-T_{1}^{4}\right) \\
& +\varepsilon_{14} \sigma_{0} \varphi_{41} F_{4}\left(T_{4}^{4}-T_{1}^{4}\right)+\frac{\lambda_{7}}{\delta_{7}} F_{7}\left(T_{7}-T_{1}\right) \\
& +\varepsilon_{1 p 4} \sigma_{0} \varphi_{41 w s} F_{41 w s}\left(T_{4}^{4}-T_{1}^{4}\right)+\varepsilon_{1 b s} \sigma_{0} \varphi_{41 b s} F_{41 b s}\left(T_{4}^{4}-T_{1}^{4}\right) \\
& +\varepsilon_{1 S c_{2}} \sigma_{0} \varepsilon_{S c_{2} 1} F_{S c_{2}}\left(T_{S c_{2}}^{4}-T_{1}^{4}\right)+\varepsilon_{1 S c_{3}} \sigma_{0} \varphi_{S c_{3} 1} F_{S c_{3}}\left(T_{S c_{3}}^{4}-T_{1}^{4}\right) \\
& +\varepsilon_{1 w s} \sigma_{0} \varphi_{51 w s} F_{5}\left(T_{5}^{4}-T_{1}^{4}\right)+\varepsilon_{1 b s 5} \sigma_{0} \varphi_{51 b s} F_{5}\left(T_{5}^{4}-T_{1}^{4}\right)=0 .
\end{aligned}
$$

Или через вариации температур, когда $\delta T_{i}=T_{i}-T_{1}$ и $\Delta T=T_{5}-T_{1}$ :

$$
\begin{aligned}
a_{2} \delta T_{2} & +a_{3} \delta T_{3}+a_{4} \delta T_{4}+a_{7} \delta T_{7}+a_{S c 2} \delta T_{S c 2} \\
& +a_{S c 3} \delta T_{S c 3}+a_{5} \Delta \boldsymbol{T}=0,
\end{aligned}
$$

где

$$
\begin{aligned}
a_{2} & =\left(T_{2}+T_{1}\right)\left(T_{2}^{2}+T_{1}^{2}\right) \varepsilon_{12} \sigma_{0} \varphi_{21} F_{2}, \\
a_{3} & =\left(T_{3}+T_{1}\right)\left(T_{3}^{2}+T\right) \varepsilon_{13} \sigma_{0} \varphi_{31} F_{3}, \\
a_{4} & =\left(T_{4}+T_{1}\right)\left(T_{4}^{2}+T_{1}^{2}\right)\left(\varepsilon_{1 w s} \sigma_{0} \varphi_{41 w s} F_{41 w s}\right. \\
& \left.+\varepsilon_{1 b s} \sigma_{0} \varphi_{41 b s} F_{41 b s}+\varepsilon_{14} \sigma_{0} \varphi_{41} F_{4}\right) T_{4}, \\
a_{7} & =\frac{\lambda_{7}}{\delta_{7}} F_{7}, \\
a_{S c_{2}} & =\left(T_{S c_{2}}+T_{1}\right)\left(T_{S c_{2}}^{2}+T_{1}^{2}\right) \varepsilon_{1 S c_{2}} \sigma_{0} \varphi_{S c_{2} 1} F_{S c_{2}}, \\
a_{S c_{3}} & =\left(T_{S c_{3}}+T_{1}\right)\left(T_{S c_{3}}^{2}+T_{1}^{2}\right) \varepsilon_{1 S c_{3}} \sigma_{0} \varphi_{S c_{3} 1} F_{S c_{3}}, \\
a_{5} & =\left(T_{5}+T_{1}\right)\left(T_{5}^{2}+T_{1}^{2}\right)\left(\varepsilon_{1 w s 5} \sigma_{0} \varphi_{51 w s} F_{5}\right. \\
& \left.+\varepsilon_{1 b s 5} \sigma_{0} \varphi_{51 b s} F_{5}\right) .
\end{aligned}
$$

Обозначения индексов: 12 - теплообмен между главным зеркалом 1 и охлаждаемым экраном 2; 13 теплообмен между главным зеркалом 1 и охлаждаемым экраном 3; 14 - теплообмен между боковой поверхностью главного зеркала 1 и медным экраном 4; 1 ws 4 теплообмен между рабочей поверхностью главного зеркала 1 и медным экраном 4; $1 b s 4$ - теплообмен между тыльной поверхностью главного зеркала 1 и медным экраном 4; $1 S c_{2}$ - теплообмен между главным зеркалом 1 и теплозащитным экраном $S c_{2} ; 1 S c_{3}$ - теплообмен между главным зеркалом 1 и теплозащитным экраном $S c_{3}$; $1 w s 5$ - теплообмен между рабочей поверхностью главного зеркала 1 и стенками камеры 5 ; $1 b s 5$ - теплообмен между тыльной поверхностью главного зеркала 1 и стенками камеры 5 .

Переход от уравнений типа (8) к уравнениям типа (9) соответствует резольвентному принципу преобразования алгебраических уравнений [9].

Система уравнений теплового баланса, составленная на основе уравнений типа (9), представляет собой линейную систему алгебраических уравнений относительно вариаций температуры от элемента к элементу, $\delta T_{i}$. В то же время относительно абсолютных значений $T_{i}$ система (9) является нелинейной с коэффициентами, зависимыми от ее решения. Для расчета $T_{i}$ предлагается использовать метод последовательных приближений [9], когда на основе решения системы (9) на каждом шаге итераций определяется $\delta T_{i}^{k}$ с последующим определением температуры каждого элемента как: $T_{i}^{k+1}=T_{i}^{k}+\delta T_{i}^{k}$, где $k$ - номер шага (итерации). Система уравнений (9) решается при помощи современных компьютерных программ. В качестве начального приближения для значений температуры $T_{i}$ принимается температура главного зеркала $T_{1}$, что соответствует технологическому циклу охлаждения коллиматора.

Важными параметрами в уравнениях (9) являются угловые коэффициенты лучистого теплообмена между элементами термовакуумной системы. Угловые коэффициенты излучения $\varphi_{i j}$ обладают следующими свойствами [7]:

a) свойство замкнутости, когда сумма долей излучения $i$-го элемента на себя и на другие поверхности, образующие замкнутую систему, равна единице, т.е.: $\sum_{k=1}^{N} \varphi_{i k}=1$;

б) свойство взаимности, означающее что для двух тел, входящих в любую замкнутую или разомкнутую систему и находящихся в состоянии радиационного теплообмена, справедливо равенство $\varphi_{i j} F_{i}=F_{j} \varphi_{j i}$;

в) свойство невогнутости (для плоских и выпуклых поверхностей), а именно угловой коэффициент „сам на себя“ равен нулю, так как в этом случае тело само на себя не излучает („не видит $\left.{ }^{6}\right): \varphi_{i i}=0$.

В каждое слагаемое в (9) входят коэффициенты, которые зависят как от температуры индивидуальных элементов, участвующих в тепловом балансе, так и от их оптических характеристик (степени черноты поверхности, коэффициента теплопроводности материала). Решение системы уравнений на основе (9) зависит от разницы абсолютных значений температуры стенок вакуумной камеры (основной источник тепла) и зеркала коллиматора, которое необходимо охладить до заданной температуры, т. е. от $\Delta T=T_{5}-T_{1}$. Конструкция термостата ограничена размерами как стенок вакуумной камеры, так 
и размерами световой зоны коллиматора, что приводит к необходимости оптимизации температурных параметров элементов термостата при заданном значении $\Delta T$. Наглядно эти ограничения иллюстрирует рис. 1, где отдельными линиями показаны углы прямой засветки зеркала, внутри которых тепловое излучение от стенок вакуумной камеры попадает на его поверхности. Здесь, $\theta_{1}$ - угол, ограниченный размером охлаждаемого экрана, $\theta_{2}$ - угол, ограниченный длиной теплозащитного экрана. Тепловому потоку внутри угла $\theta_{2}$ соответствуют, например, слагаемые в (9) с коэффициентом $a_{5}=\left(T_{5}+\right.$ $\left.+T_{1}\right)\left(T_{5}^{2}+T_{1}^{2}\right)\left(\varepsilon_{1 w s 5} \sigma_{0} \varphi_{51 w s} F_{5}+\varepsilon_{1 b s 5} \sigma_{0} \varphi_{51 b s} F_{5}\right)$, в которую входят соответствующие угловые коэффициенты излучения $\varphi_{51 w s}$ и $\varphi_{51 b s}$. Здесь $F_{5}-$ часть поверхности вакуумной камеры, излучение с которой попадает на зеркало. Влияние угловых коэффициентов излучения на температурные параметры требует специального рассмотрения, так как в термостате используются цилиндрические экраны для охлаждения плоского зеркала.

\section{3. Угловые коэффициенты теплообмена между зеркалом и цилиндрическими экранами}

Рассмотрим зависимость угловых коэффициентов излучения для главного зеркала с цилиндрическими экранами от геометрических параметров поверхностей этих элементов и расстояния между ними. Для этого введем эффективный (тепловой) радиус общей поверхности „зеркало + подставка“ так, что:

$$
R_{1}=\sqrt{R_{0}^{2}+\frac{F_{\text {sup }}}{\pi}} .
$$

В этом случае угловые коэффициенты излучения $\varphi_{12}$ и $\varphi_{13}$ рассчитываются по формулам [2] в виде разности угловых коэффициентов теплообмена между зеркалом и двумя параллельными соосными дисками, расположенными друг относительно друга на расстоянии длины бленды, т.е.

$$
\begin{aligned}
& \varphi_{12}=\frac{1}{2 R_{1}^{2}}\left(l^{2}+R_{1}^{2}+R_{2}^{2}-\sqrt{\left(l^{2}+R_{1}^{2}+R_{2}^{2}\right)^{2}-4 R_{1}^{2} R_{2}^{2}}\right) \\
& -\frac{1}{2 R_{1}^{2}}\left(\left(L_{2}+l\right)^{2}+R_{1}^{2}+R_{2}^{2}\right. \\
& \left.-\sqrt{\left(\left(L_{2}+l\right)^{2}+R_{1}^{2}+R_{2}^{2}\right)^{2}-4 R_{1}^{2} R_{2}^{2}}\right) \\
& =\frac{1}{2}\left(\sqrt{\left(\frac{l^{2}}{R_{1}^{2}}+\frac{2 L_{2} l}{R_{1}^{2}}+\frac{L_{2}^{2}}{R_{1}^{2}}+1+\frac{R_{2}^{2}}{R_{1}^{2}}\right)^{2}-4 \frac{R_{2}^{2}}{R_{1}^{2}}}\right. \\
& -\sqrt{\left.\left(\frac{l^{2}}{R_{1}^{2}}+1+\frac{R_{2}^{2}}{R_{1}^{2}}\right)^{2}-4 \frac{R_{2}^{2}}{R_{1}^{2}}-\left(\frac{2 L_{2} l}{R_{1}^{2}}+\frac{L_{2}^{2}}{R_{1}^{2}}\right)\right) .}
\end{aligned}
$$

Здесь $R_{2}$ - радиус цилиндрического экрана, $L_{2}-$ длина цилиндрического экрана, $l-$ расстояние между цилиндрическим экраном и главным зеркалом.

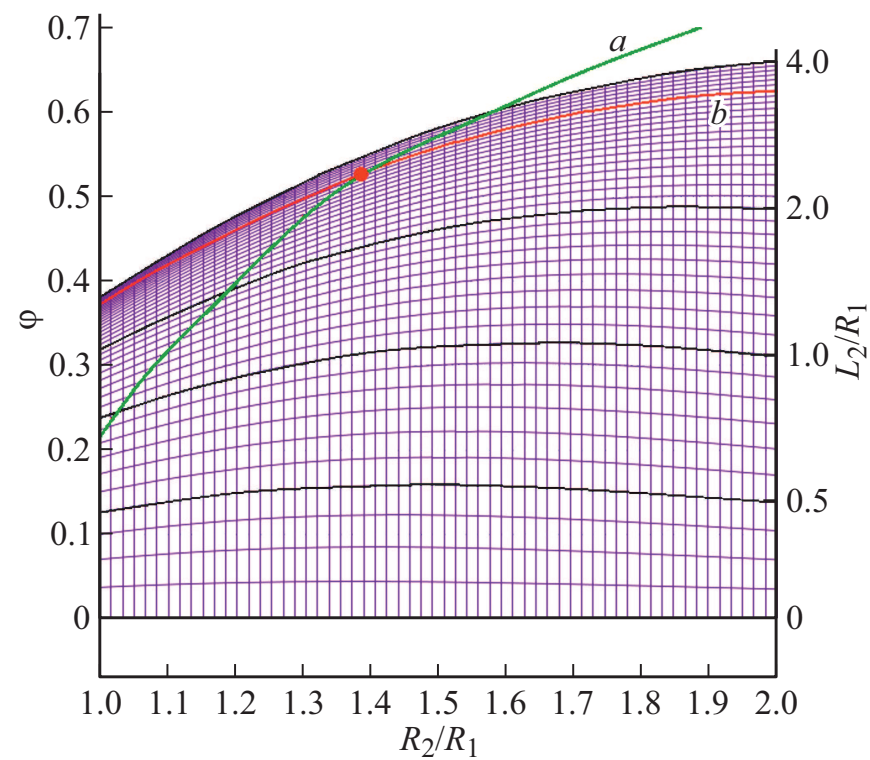

Рис. 2. Зависимость углового коэффициента теплового излучения от длины и радиуса цилиндрического экрана 2 (линия $a$ соответствует экрану 2 рис. 1 , линия $b$ показывает ограничения по световой зоне). $L_{2} / R_{1}-$ безразмерная величина длины цилиндрического экрана $2, R_{2} / R_{1}$ - безразмерная величина радиуса цилиндрического экрана 2.

На рис. 2 приведен конкретный пример зависимости углового коэффициента излучения $\varphi_{12}$ от длины и радиуса экрана 2 в виде безразмерных параметров, определяемых по отношению к радиусу зеркала. Видим, что с увеличением длины экрана увеличивается значение углового коэффициента излучения, при этом его максимум сдвигается вправо, когда радиус увеличивается. В варианте схемы термостата (рис. 1) геометрические параметры цилиндрического экрана 2 (точка рис. 7) выбраны таким образом, чтобы экран не перекрывал световую зону коллиматора при максимальном значении $\varphi_{12}$. Изменение его геометрических параметров с учетом световой зоны коллиматора соответствует линии $b$ на рис. 2. Видно, что абсолютный максимум углового коэффициента излучения $\varphi_{12}$ в данной схеме не достигается из-за ограниченного диаметра вакуумной камеры и размера световой зоны коллиматора.

Другой пример выбора $\varphi_{i j}$ представлен на рис. 3 для цилиндрического экрана 3 , который расположен ближе к зеркалу, чем экран 2. В этом случае максимум углового коэффициента излучения $\varphi_{13}$ наблюдается при небольшом превышении внутреннего радиуса экрана над радиусом зеркала. Из рис. 3 следует, что в данной конструкции термостата можно подобрать геометрические параметры цилиндрического экрана 3 таким образом, чтобы теплообмен между ним и главным зеркалом был бы максимальным.

Одним из принципиальных требований при охлаждении зеркал коллиматора является обеспечение однородности распределения температуры как по оси зеркала, 


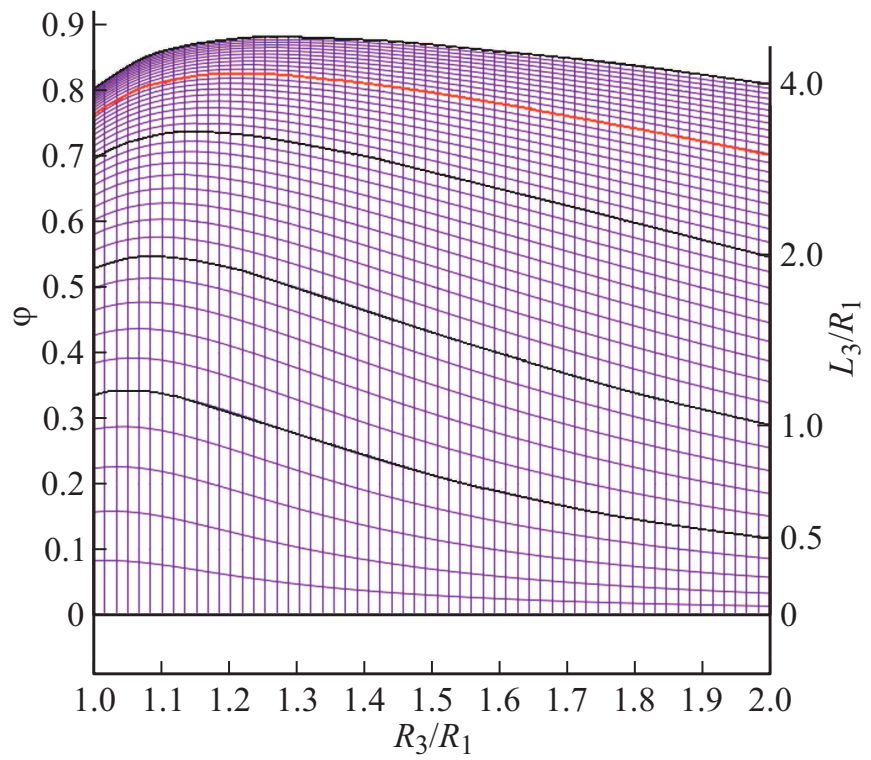

Рис. 3. Зависимость углового коэффициента теплового излучения от длины и радиуса экрана $3 . L_{3} / R_{1}-$ безразмерная величина длины цилиндрического экрана $3, R_{3} / R_{1}$ - безразмерная величина радиуса цилиндрического экрана 3.

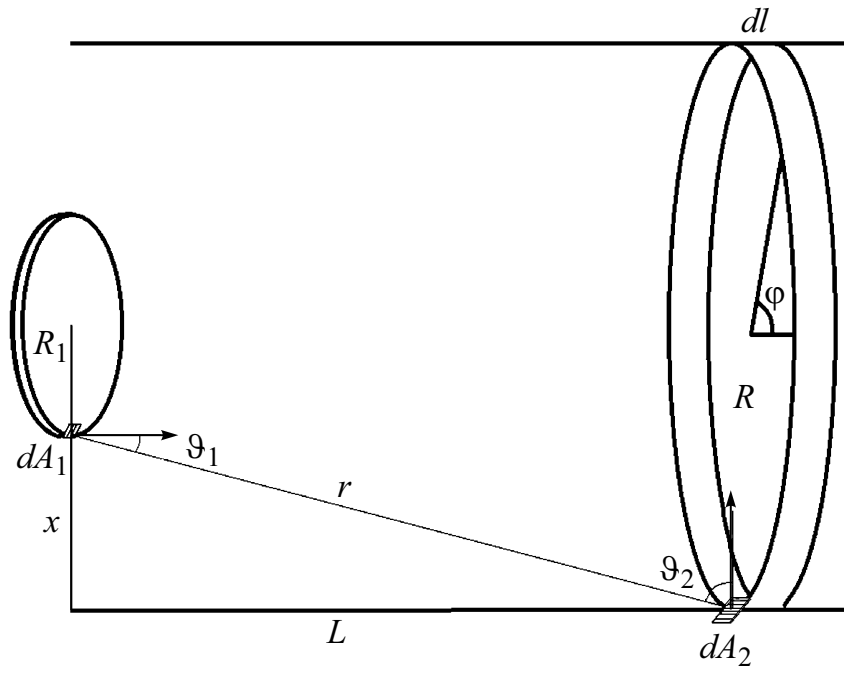

Рис. 4. Элемент плоскости на поверхности круглого диска (зеркало) и элемент площадки на внутренней стороне круглого цилиндра.

так и по его радиусу, чтобы устранить возможные термоаберрации зеркальной поверхности. Если условие осевой однородности распределения температуры входит в виде специального уравнения в систему уравнений на основе (9), то на радиальное распределение может оказать влияние неравномерность распределения локальных угловых коэффициентов излучения по радиусу зеркала.

Определим это влияние, используя пример теплообмена излучением между двумя произвольно расположенными поверхностями $A_{1}$ и $A_{2}$ конечных размеров (рис. 4), температуры которых соответственно равны $T_{1}$ и $T_{2}$ [6]. Возьмем элементарную площадку $d A_{1}$ на поверхности главного зеркала, с которого лучистая энергия попадает на площадку $d A_{2}$ на стенке цилиндрического экрана на расстоянии $r$. Тепловой поток с площадки $d A 1$ равен

$$
d I_{1}=\frac{I}{\pi} \cos \theta d \omega d A_{1},
$$

где $I_{1}-$ плотность (интенсивность) теплового излучения. Если среди всех возможных направлений $\theta$ выбрать направление на площадку $d A_{2}$, то $\theta=\theta_{1}$, тогда и тепловой поток с площадки $d A_{1}$ на $d A_{2}$ будет равен

$$
d I_{1 \rightarrow 2}=\frac{d A_{1} d A_{2} \cos \theta_{1} \cos \theta_{2}}{r^{2}} \frac{M}{\pi} .
$$

Локальный угловой коэффициент излучения $d \varphi_{d A_{1}, A_{2}} \mathrm{c}$ площадки $d A_{1}$ на всю поверхность $d A_{2}$ соответственно можно определить как:

$$
d \varphi_{d A_{1}, A_{2}}=\int_{A_{2}} \frac{\cos \theta_{1} \cos \theta_{2} d A_{2}}{\pi r^{2}} .
$$

Параметры, входящие в формулу (14), определяются следующим образом:

$$
x=\sqrt{\left(R_{1}\right)^{2}+(R)^{2}-2 R_{1} R \cos \varphi}
$$

(по теореме косинусов), $\cos \theta_{1}=\frac{L+l}{r} ; \quad \cos \theta_{2}=\frac{x}{r}$, $d A_{2}=R d \varphi d l$ и $r=\sqrt{(L+l)^{2}+x^{2}}$, где $R_{1}$ - радиус зеркала коллиматора, $R$ - радиус внутренних стенок экрана, $L-$ расстояние от $(\cdot) A_{1}$ на поверхности главного зеркала до элемента площадки $d A_{2}$ (в виде ленты), $r$ - расстояние от элемента площадки $d A_{1}$ на поверхности зеркала до элемента площадки $d A_{2}$ на экране, $x$ - расстояние от элемента площадки на поверхности зеркала до стенки экрана по нормали к ней.

Тогда угловой коэффициент излучения с элементарной площадки на поверхности главного зеркала на ленточную поверхность с радиусом $R$ и шириной $l$ равен:

$$
\begin{aligned}
& d \varphi_{d A_{1}, A_{2}}= \\
& \int_{0}^{2 \pi} \int_{0}^{l} \frac{\left(\frac{L}{R_{1}}+l\right) \sqrt{1+\left(\frac{R}{R_{1}}\right)^{2}-2 \frac{R}{R_{1}} \cos \varphi \frac{R}{R_{1}}}}{\pi\left(\left(\frac{L}{R_{1}}+l\right)^{2}+1+\left(\frac{R}{R_{1}}\right)^{2}-2 \frac{R}{R_{1}} \cos \varphi\right)^{2}} d \varphi d l .
\end{aligned}
$$

На рис. 5 представлены графики изменения углового коэффициента теплового излучения (15) вдоль радиуса главного зеркала в зависимости от расстояния (в безразмерном виде) от зеркала до цилиндрической ленты.

Наибольшее изменение значений углового коэффициента излучения по поверхности зеркала наблюдается на расстоянии $L / R_{1}=0-0.9$ от зеркала (в данной схеме термостата). При большем расстоянии их изменение 

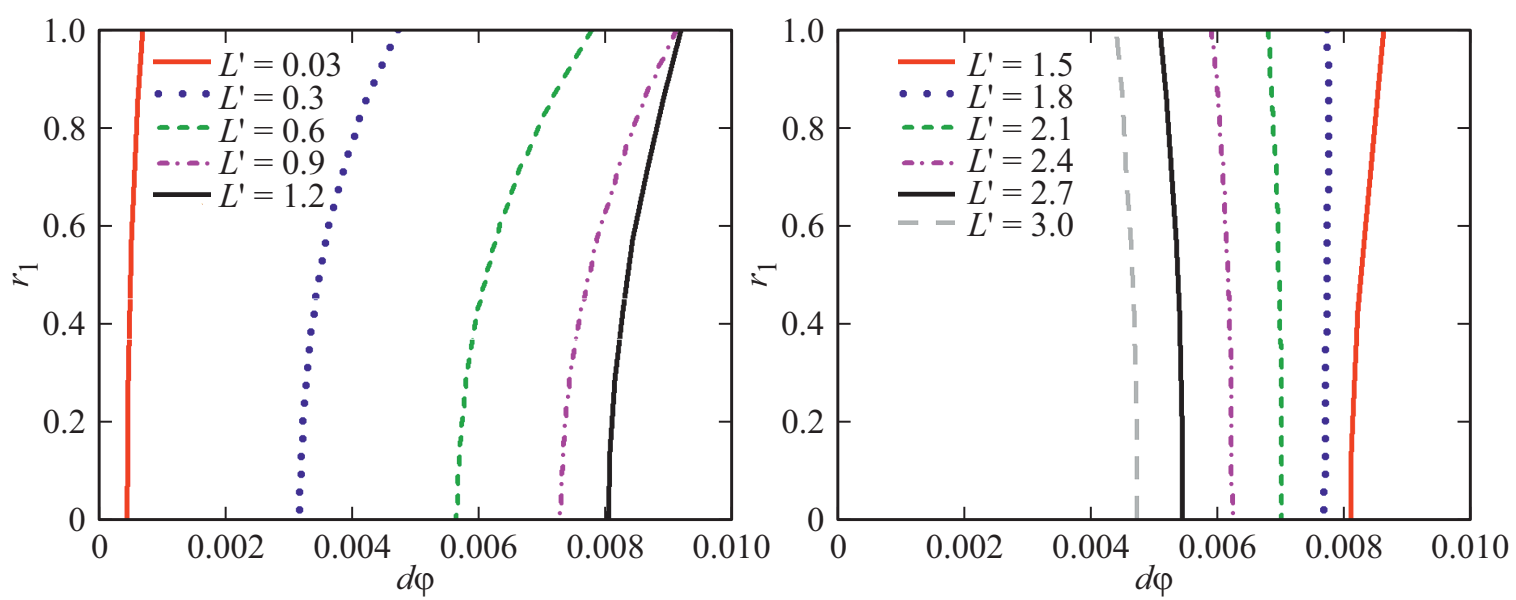

Рис. 5. Локальные угловые коэффициенты теплового излучения зеркала для различных расстояний до поверхности цилиндрического экрана, $r_{1}$ - изменение радиуса зеркала, $d \varphi-$ локальный угловой коэффициент излучения.

незначительно. При этом если это расстояние меньше 1.5 , то в центре зеркала значение углового коэффициента излучения меньше, чем на его краях.

По характеру изменения кривых можно сказать, что величина теплового потока в центре зеркала будет меньше, чем на его краях. Так же можно заметить, что значение угловых коэффициентов излучения увеличивается при увеличении расстояния от экрана до зеркала, но начиная с некоторого расстояния $L / R_{1}=1.2$, они уменьшаются, и в центре зеркала температура меньше, чем на его краях. Этот факт показывает, что можно обеспечить выравнивание распределения температуры по поверхности зеркала за счет комбинирования угловых коэффициентов излучения и температуры охлаждаемого экрана.

\section{4. Оптимизация теплофизических параметров в тепловой модели}

Для иллюстрации возможности оптимизации температурных и теплофизических параметров термостата с использованием разработанного метода на рис. 6 приведены результаты расчетов зависимости температуры цилиндрических охлаждаемых экранов 2 и 3 от температуры зеркала по схеме термостата, показанной на рис. 1 . Значками (треугольники и кружки) на рисунке обозначены точные решения системы уравнений, составленных на базе уравнений типа (9), а линиями (сплошными и штриховые) обозначены линейные приближения к точному решению для двух вариантов конструкции термостата, отличающихся степенью черноты охлаждаемых экранов.

Следует отметить, что в принятой схеме термостата „линейное“ решение дает завышенные значения температуры охлаждаемых экранов. Важно также, что при значении температуры зеркала $T_{1}^{*}$ ниже $193 \mathrm{~K}$ нет точного решения, т.е. охлаждение зеркала ниже $T_{1}^{*}$ только

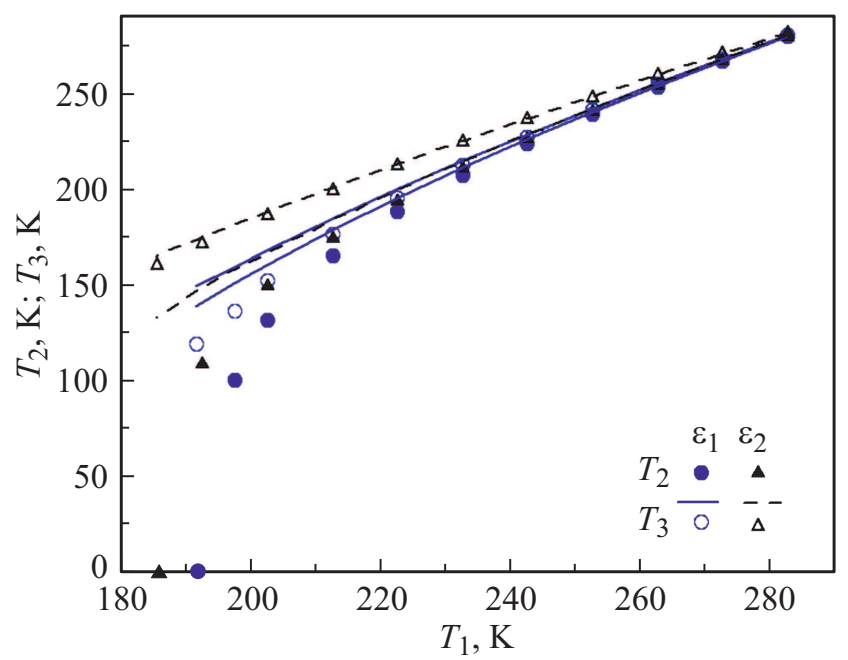

Рис. 6. Изменение температурных параметров охлаждаемых цилиндрических экранов в зависимости от температуры зеркала $\left(\varepsilon_{1}<\varepsilon_{2}\right), T_{i}$ - температура $i$-го элемента.

с помощью двух охлаждаемых экранов в данной схеме термостата обеспечить невозможно.

В качестве доказательства сходимости метода последовательных итераций, который используется в расчетах, в табл. 1 приведены результаты расчета баланса тепловых потоков на каждом элементе термостата, полученные для каждой точки графиков рис. 6.

Из табл. 1 следует, что в случае „установившегося“ решения системы уравнений (9), с достаточно большой точностью (погрешность на уровне компьютерных ограничений) соблюдаются условия теплового баланса для каждого элемента термостата.

Ограниченность решения от $T_{1}$ проявляется из-за большой тепловой нагрузки на охлаждаемые цилиндрические экраны, которая возрастает с увеличением разности между температурой стенок камеры и зеркала. 
Таблица 1. Баланс тепловых потоков на элементах термостата и коллиматора

\begin{tabular}{c|c|c|c|c|c|c}
\hline$T_{1}, \mathrm{~K}$ & $Q_{1}, \mathrm{~W}$ & $Q_{4}, \mathrm{~W}$ & $Q_{7}, \mathrm{~W}$ & $Q_{8}, \mathrm{~W}$ & $Q_{9}, \mathrm{~W}$ & $Q_{6}, \mathrm{~W}$ \\
\hline 283 & $4.1 \cdot 10^{-11}$ & 0 & $-4.1 \cdot 10^{-11}$ & $4.4 \cdot 10^{-12}$ & $-9.9 \cdot 10^{-14}$ & $-1.9 \cdot 10^{-14}$ \\
\hline 273 & $7.1 \cdot 10^{-12}$ & $-5.3 \cdot 10^{-15}$ & $-7.1 \cdot 10^{-12}$ & $3.0 \cdot 10^{-12}$ & $-1.0 \cdot 10^{-13}$ & $-1.6 \cdot 10^{-14}$ \\
\hline 263 & $3.3 \cdot 10^{-11}$ & $3.4 \cdot 10^{-15}$ & $-3.3 \cdot 10^{-11}$ & $-4.4 \cdot 10^{-12}$ & $1.4 \cdot 10^{-12}$ & $-4.0 \cdot 10^{-12}$ \\
\hline 253 & $4.7 \cdot 10^{-12}$ & $2.8 \cdot 10^{-15}$ & $-4.7 \cdot 10^{-12}$ & $6.2 \cdot 10^{-13}$ & $1.3 \cdot 10^{-13}$ & $-1.9 \cdot 10^{-14}$ \\
\hline 243 & $-1.5 \cdot 10^{-11}$ & $-3.3 \cdot 10^{-15}$ & $1.5 \cdot 10^{-11}$ & $2.0 \cdot 10^{-13}$ & $-1.8 \cdot 10^{-14}$ & $-3.4 \cdot 10^{-14}$ \\
\hline 233 & $4.1 \cdot 10^{-12}$ & $1.1 \cdot 10^{-15}$ & $-4.1 \cdot 10^{-12}$ & $1.5 \cdot 10^{-12}$ & $1.8 \cdot 10^{-14}$ & $-2.0 \cdot 10^{-14}$ \\
\hline 223 & $-4.9 \cdot 10^{-12}$ & 0 & $4.9 \cdot 10^{-12}$ & $1.0 \cdot 10^{-12}$ & $5.7 \cdot 10^{-14}$ & $-2.4 \cdot 10^{-14}$ \\
\hline 213 & $1.0 \cdot 10^{-11}$ & $1.4 \cdot 10^{-15}$ & $-1.0 \cdot 10^{-11}$ & $2.1 \cdot 10^{-12}$ & $-6.4 \cdot 10^{-14}$ & $2.1 \cdot 10^{-13}$ \\
\hline 203 & $-2.7 \cdot 10^{-12}$ & $-1.2 \cdot 10^{-14}$ & $2.6 \cdot 10^{-12}$ & $4.2 \cdot 10^{-14}$ & $-6.4 \cdot 10^{-14}$ & $3.4 \cdot 10^{-14}$ \\
\hline 198 & $5.8 \cdot 10^{-13}$ & $-9.0 \cdot 10^{-14}$ & $-1.1 \cdot 10^{-12}$ & $-1.3 \cdot 10^{-12}$ & $8.5 \cdot 10^{-14}$ & $-5.6 \cdot 10^{-13}$
\end{tabular}

При ме чан ие: $Q_{i}-$ соответствует формуле $(5)$ для $i$-го элемента.

Таблица 2. Температурные параметры элементов термостата при охлаждении зеркала коллиматора

\begin{tabular}{c|c|c|c|c|c|c}
\hline$T_{1}, \mathrm{~K}$ & $T_{2}, \mathrm{~K}$ & $T_{3}, \mathrm{~K}$ & $T_{S c_{1}}, \mathrm{~K}$ & $T_{S c_{2}}, \mathrm{~K}$ & $T_{S c_{3}}, \mathrm{~K}$ & $T_{S c_{10}}, \mathrm{~K}$ \\
\hline 283 & 282.6 & 282.4 & 284.2 & 283.6 & 284.1 & 289 \\
\hline 273 & 272.2 & 271.8 & 275.5 & 274.3 & 275.3 & 285.3 \\
\hline 263 & 261.7 & 261.1 & 266.9 & 265.1 & 266.6 & 281.9 \\
\hline 253 & 251.1 & 250.3 & 258.4 & 256 & 258.1 & 278.7 \\
\hline 243 & 240.5 & 239.3 & 250.1 & 246.9 & 249.8 & 275.7 \\
\hline 233 & 229.7 & 228.2 & 241.9 & 238 & 241.6 & 273 \\
\hline 223 & 218.8 & 216.8 & 233.9 & 229.3 & 233.7 & 270.6 \\
\hline 213 & 207.7 & 205.2 & 226.2 & 220.8 & 226 & 268.4 \\
\hline 203 & 196.4 & 193.2 & 218.6 & 212.4 & 218.7 & 266.5 \\
\hline 193 & 184.8 & 180.7 & 211.4 & 204.4 & 211.7 & 264.8 \\
\hline 183 & 172.8 & 167.5 & 204.4 & 196.6 & 205.2 & 263.3 \\
\hline 173 & 160.1 & 153.1 & 197.9 & 189.2 & 199 & 262 \\
\hline 163 & 146.5 & 136.7 & 191.7 & 182.2 & 193.4 & 260.9 \\
\hline 153 & 131.2 & 116.3 & 186.1 & 175.8 & 188.3 & 260 \\
\hline 143 & 112.8 & 82.5 & 180.9 & 169.8 & 183.8 & 259.2
\end{tabular}

Примеч ани е: $T_{i}-$ температура $i$-го элемента.

Эта нагрузка учитывается соответствующими членами в уравнениях теплового баланса как для зеркала (см. выделенные слагаемые в первых двух уравнениях типа (9)), так и в уравнениях теплового баланса теплозащитных экранов. Уменьшить тепловую нагрузку на охлаждаемые экраны можно изменением конструкции теплозащитных экранов, увеличив как их количество, так и длину вдоль оптической оси коллиматора.

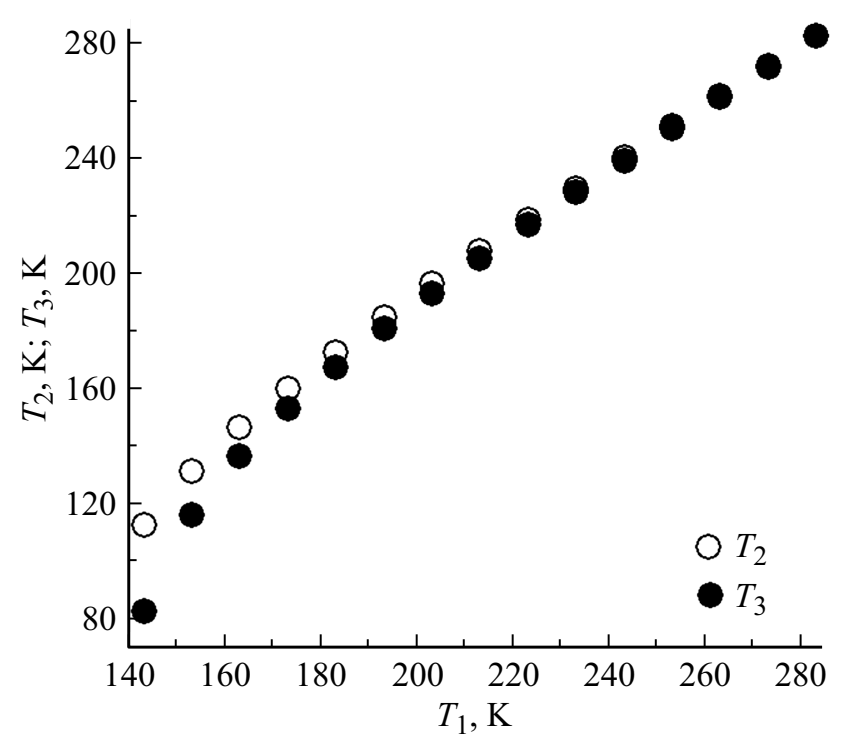

Рис. 7. Изменение температуры охлаждаемых экранов в условиях теплового равновесия, $T_{i}$ - температура $i$-го элемента.

В табл. 2 приведены результаты расчетов охлаждения зеркала коллиматора с использованием схемы термостата, в которую вставлен дополнительный теплозащитный экран с максимальной длиной, ограниченной световой зоной коллиматора. Здесь также приведены значения температуры всех элементов термостата при последовательном понижении температуры зеркала коллиматора. Температура стенок камеры считалась постоянной и равной $20^{\circ} \mathrm{C}$.

Рис. 7 иллюстрирует зависимости температуры охлаждаемых экранов $\left(T_{2}, T_{3}\right)$ в условиях теплового равновесия при изменении температуры главного зеркала для схемы термостата с дополнительным теплозащитным экраном. 
Применение дополнительных теплозащитных экранов позволяет существенно увеличить температурный диапазон главного зеркала коллиматора при ограниченной холодильной мощности криогенной системы. Следует также отметить, что в этом варианте термостата значения температуры охлаждаемых экранов отличаются от температуры главного зеркала незначительно (в пределах $10 \%)$.

Полученные результаты расчетов достаточно наглядно иллюстрируют возможности разработанного метода расчета термовакуумных систем регулирования и стабилизации температуры в зависимости как от конструкций элементов термостата, так и от их оптических (степень черноты поверхности, которая зависит от материала) и теплофизических характеристик. Необходимым условием использования данного метода при этом является корректное определение угловых коэффициентов обмена лучистой энергией в системе „термостат-коллиматор“.

\section{Заключение}

В результате проведенных исследований обоснована теплофизическая модель термовакуумного регулирования и стабилизации элементов коллиматора в ограниченном вакуумном объеме. Модель основана на принципе теплового равновесия в системе „коллиматор-термостат“ с привлечением уравнений лучистого теплообмена между элементами системы, включая возможные процессы передачи тепла за счет теплопроводности между ними. Особенностью модели является обеспечение условий минимизации термоаберраций зеркал коллиматора при его охлаждении.

Тепловая модель термостата сводится к системе нелинейных (4 степени) алгебраических уравнений. Для расчета и оптимизации температурных параметров термостата при заданной температуре главного зеркала коллиматора используется метод простой итерации (метод последовательных приближений) для уравнений, составленных относительно вариаций температуры элементов термостата относительно температуры охлаждаемого зеркала (с понижением степени нелинейности уравнений).

Показана возможность минимизации радиационных потоков ИК излучения при оптимизации конструкции термостата, его охлаждаемых элементов (цилиндрических экранов), а также количества теплозащитных экранов. Предложенная функциональная схема термостата может быть использована для снижения температуры радиационного фона в термовакуумных стендах для испытаний ИК ОЭС.

\section{Конфликт интересов}

Авторы заявляют, что у них нет конфликта интересов.

\section{Список литературы}

[1] С.В. Любарский, Л.Ш. Олейников, В.Б. Григорьев, М.М. Чулков, А.А. Шнырев. Оптич. журн., 64 (8), 20 (1997).

[2] Р. Зигель, Дж. Хауэлл. Теплообмен излучением (Мир, М., 1975)

[3] Л.Ш. Олейников. Методы и средства стабилизащии оптических параметров криотелескопов космического базирования и наземных имитационно-испытательных комплексов. Диссертация на соискание ученой степени д.т.н. С.-Петербург, 2004.

[4] J.A. Clinea, J. Quennevillea, R.S. Taylora, T. Deschenesa, M. Braunsteina, H. Legnerb, B.D. Green. Proceedings of 7-th Nanophotonics and Microphotonics foe Space Environments Conf., 2013. P. 3012-3018. http://dx.doi.org/10.1117/12.2024371

[5] В.М. Фокин, Г.П. Бойков, Ю.В. Видин. Основы технической теплофизики (Машиностроение, М., 2004)

[6] J.H. Lienhard IV, J.H. Lienhard V. A heat transfer textbook (Phlogiston Press, 2019), p. 772.

[7] Г.Л. Саксаганский. Молекулярные потоки в сложных вакуумных структурах (Атомиздат, М., 1980)

[8] Л.С. Полак. Вариационные принциипы механики: Их развитие и применения в физике (URSS, M., 2017)

[9] Дж. Ортега, В. Рейнболт. Итерационные методы решения нелинейных систем уравнений со многими неизвестными (Мир, М., 1975) 